OPEN ACCESS

Edited by:

Mario Caba,

Universidad Veracruzana, Mexico

Reviewed by:

Ilia N. Karatsoreos,

Washington State University,

United States

Raul Aguilar-Roblero,

Universidad Nacional Autónoma de México, Mexico

*Correspondence:

Antonio A. Nunez

nunez@msu.edu

Specialty section:

This article was submitted to

Neuroendocrine Science,

a section of the journal

Frontiers in Endocrinology

Received: 04 January 2018

Accepted: 19 February 2018

Published: 07 March 2018

Citation:

Nunez AA, Yan L and Smale L (2018)

The Cost of Activity during the Rest

Phase: Animal Models and

Theoretical Perspectives.

Front. Endocrinol. 9:72.

doi: 10.3389/fendo.2018.00072

\section{The Cost of Activity during the Rest Phase: Animal Models and Theoretical Perspectives}

\author{
Antonio A. Nunez ${ }^{1 *}$, Lily Yan ${ }^{1}$ and Laura Smale ${ }^{1,2}$ \\ ${ }^{1}$ Department of Psychology and Neuroscience Program, Michigan State University, East Lansing, MI, United States, \\ ${ }^{2}$ Department of Integrative Biology, Michigan State University, East Lansing, MI, United States
}

For humans, activity during the night is correlated with multiple pathologies that may reflect a lack of harmony among components of the circadian system; however, it remains difficult to identify causal links between nocturnal activity and different pathologies based on the data available from epidemiological studies. Animal models that use forced activity or timed sleep deprivation provide evidence of circadian disruptions that may be at the core of the health risks faced by human night and shift workers. One valuable insight from that work is the importance of changes in the distribution of food intake as a cause of metabolic imbalances associated with activity during the natural rest phase. Limitations of those models stem from the use of only nocturnal laboratory rodents and the fact that they do not replicate situations in which humans engage in work with high cognitive demands or engage voluntarily in nocturnal activity (i.e., human eveningness). Temporal niche switches by rodents have been observed in the wild and interpreted as adaptive responses to energetic challenges, but possible negative outcomes, similar to those associated with human eveningness, have not been systematically studied. Species in which a proportion of animals shows a switch from a day-active to a night-active (e.g., grass rats) when given access to running wheels provide a unique opportunity to model human eveningness in a diurnal rodent. In particular, the mosaic of phases of brain oscillators in night-active grass rats may provide clues about the circadian challenges faced by humans who show voluntary nocturnal wakefulness.

Keywords: shift work, circadian rhythms, temporal niche, eveningness, grass rats

\section{HUMAN NIGHT WORK}

Our contemporary global society has created demands that require many of us to be active during the natural rest phase of our species, the night. This is exemplified by increasing number of individuals who work nights and thus are awake and engaged with the environment at least for part of the time normally dominated by human sleep. There is a rich, albeit mostly correlational, literature linking night and shift work to a multitude of pathologies including higher risk of cancer (1), metabolic syndrome (2), hypertension (3), cognitive deficits (4), and female infertility (5), among other health and behavioral problems. Many of these negative outcomes may stem from a lack of harmony among different components of the circadian system (6).

The circadian system consists of a principal oscillator located in the hypothalamic suprachiasmatic nucleus $(\mathrm{SCN})(7,8)$ that entrains to the light-dark cycle via direct retinal projections from melanopsin-containing retinal ganglion cells (9). Outputs of the SCN serve to synchronize a multitude of extra-SCN oscillators in the brain and in the peripheral organs (10). Night or shift 
work is associated with exposure to environmental influences that challenge the temporal regulation of many behavioral and physiological functions. When undisturbed, the resulting daily rhythms are kept at optimal phase relations among themselves and synchronized (entrained) to the 24-h day-night cycle by the circadian system. Some of the challenges faced by the circadian system of night workers include exposure to nocturnal light, activity during the natural sleep period, and food consumption during the rest phase of the cycle. It is very likely that many of the health and behavioral problems of night or shift workers stem from circadian disruptions resulting from external (light) and internal (metabolic signals; release of neurotransmitters) stimuli that interfere with, or override, circadian signals emanating from the $\operatorname{SCN}(6,11)$. However, it is difficult to draw conclusions about causality from epidemiological studies with humans. A number of animal models have been developed to circumvent those limitations.

\section{ANIMAL MODELS OF NIGHT WORK: INSIGHTS AND CHALLENGES}

Animal models of shift work have used almost exclusively laboratory rodents (see Ref. (12) for a review) that are forced to be active during their rest phase by placing them in rotating wheels for several hours every day (13-15) or alternatively, by keeping them awake during the rest phase using gentle stimulation whenever the animal gives signs of falling sleep (16). Forced activity for $8 \mathrm{~h}$ /day during the normal rest phase of laboratory rats, sustained for five consecutive days per week, results in increased abdominal fat accumulation and the display of several indicators of metabolic syndrome, including impaired glucose tolerance (13). These animals also shift their food intake to the light period $(13,14)$ and show reduced general activity as well as a reduced activityrhythm amplitude on days off from the forced activity regime (17). Interestingly, providing food only during the normal active (dark) phase prevents many of the effects of forced activity during the light phase (15), and at least in studies using rats, restricting feeding to the light phase in otherwise undisturbed animals mimics the effects of the forced activity manipulation (15). A different study that used a forced activity paradigm similar to that of experiments reporting increases in body weight and adiposity, surprisingly found a reduced body weight in the shiftworking rats (18). Differences in housing conditions or stress level of the animals could be responsible for the different outcomes, but of note is the observation that in these shift-work animals that lost weight, the amount and distribution of activity on days off did not differ from those of control animals not exposed to the forced activity regime. Thus, both changes in energy expenditure and the emergence of day-time feeding appear to contribute to the metabolic effects of forced activity during the normal rest phase of nocturnal laboratory rats.

There is ample evidence that sleep deprivation per se can negatively affect metabolism and energy balance $(19,20)$. Experiments in which chronic timed sleep restriction, with opportunity for sleep recovery within the $24-\mathrm{h}$ period, are most relevant as animal models of shift work. Several studies using mice have reported metabolic deficits including abnormal glucose and lipid metabolism when the animals are deprived of sleep during the first $6 \mathrm{~h}$ of the night for two blocks of 5 days separated by 2 days of ad lib sleep $(16,21)$. Restricting feeding to the night prevented these metabolic effects (16); however, different from what was reported for rats, restricting feeding to the light phase without sleep restriction did not result in metabolic anomalies (16). Also different from most of the forced activity work with rats, sleep restriction did not affect body weight in mice (16).

Although studies using forced timed activity or timed sleep restriction provide causal links between the human experience of night shift work and circadian, metabolic, and energy disruptions reported for these workers, they have some clear limitations. First, the use of nocturnal laboratory rodents poses questions about how generalizable the findings are to diurnal species such as ours. Also, even within nocturnal rodents, the limited data hint at possible differences between laboratory rats and mice (12), some of which likely stem from differences in body size and how that affects responses to metabolic challenges. Using a wider range of species, including day-active (DA) ones, would add significantly to the value of animal models of human shift work that use forced activity or timed sleep restriction.

\section{HUMAN EVENINGNESS}

Although humans are clearly diurnal, many of us become active during our normal rest phase, the night. This change in phase preference is not limited to those engaged in shift or night work. For example, many young adults shift their activity phase and display what is known as "eveningness," which involves being active during a large proportion of the night. There is compelling evidence that in humans, voluntary shifts to a nocturnal activity profile result in substantial negative outcomes, including eating disorders (22), diabetes, and metabolic syndrome (23). Further, eating at the inappropriate phase has been linked to obesity in humans and animals $(24,25)$. These problems have clear negative impacts on the human capital of society. The animal models that simulate human shift work using forced activity or timed sleep deprivation (see above) are not ideal to study the consequences of the apparent voluntary temporal niche switch of human eveningness. For example, the expression of Fos protein in the brain of grass rats (Arvicanthis niloticus) is remarkably different if the animals are forcibly kept awake at night compared to when they show unconstrained night wakefulness $(26,27)$ (more about this animal model below). Interestingly, shifts in the balance between day/night activity, like those seen when eveningness emerges in teenagers, have been reported for other mammalian species, both in the field and in the laboratory (28-30) and thus represent potential models for understanding the causal links between human voluntary nocturnal activity and the negative outcomes associated with it.

\section{MAMMALIAN TEMPORAL NICHE SWITCHES}

Mice, which are strictly nocturnal in standard laboratory conditions, can switch to diurnality when observed under more natural conditions for extended periods of time (31). An influential 
hypothesis to account for these switches by mice postulates that they occur in response to energy challenges (32). Specifically, this perspective suggests that situations in which animals experience negative energy balance favor the display of a diurnal phenotype (33). Laboratory work testing this hypothesis has used a "work for food" paradigm in which mice get food only if they run in a wheel, thus emulating the foraging demands of the wild (34). The workload to obtain a particular amount of food is manipulated to resemble environments with different densities of resources. Under those conditions, increasing the workload induces a phase advance of the activity (or work) rhythm, such that normally nocturnal mice show predominantly diurnal activity (32). Reduced ambient temperature, while kept with ad lib food availability, also induces a shift to diurnal activity, and enhanced workload and low ambient temperature challenges have additive effects with respect to this temporal niche switching in mice (35). The change in the phase preference for the display of activity when mice experience a negative energy balance is not accompanied by a shift in the phase of the SCN oscillator, but peripheral oscillators in the liver and adrenal gland show a phase that more closely resembles that of diurnal mammals (35).

The thermoenergetic hypothesis advanced by Hut and coworkers $(30,32)$ suggests that diurnality emerges in rodents to reduce energy needs, since days are warmer than nights. While this hypothesis promotes the adaptive value (32) of the temporal niche switch (31), there may also be costs, as activity during the natural rest phase of the mice was accompanied by changes in synchrony of internal rhythms. Specifically, peripheral oscillators shifted their phase angles with respect to the SCN and likely with respect to the melatonin rhythm, which remains nocturnal in other models of temporal niche switching $(29,36)$.

\section{TEMPORAL NICHE SWITCHES IN DIURNAL SPECIES: THE GRASS RAT AS A MODEL}

There are examples of species that are diurnal in the field, but that switch to a nocturnal activity profile in the laboratory (37). Based on the thermoenergetic hypothesis, these observations suggests that these animals may be exposed to energetic challenges in the wild that favor diurnality and that do not exist in the laboratory. More interesting from the perspective of developing a model for human eveningness are species, e.g., Nile grass rats (28) and Octodon degus (29), sometimes referred to as dual-phasing animals (29), which are diurnal in the field, and also under standard laboratory conditions, but can show either diurnal or nocturnal phenotype when given access to running wheels (28).

For over 20 years, our group has been developing the grass rat as a diurnal mammalian animal model to study the circadian system (38). To go with their diurnal life style, grass rats feature an abundance of retinal cones (39) and an optic tectum that, relative to body size, is four times the volume of that of laboratory rats (40). Although the phase of the SCN oscillator with respect to the light-dark cycle is similar to that of nocturnal rodents (41), brain and peripheral extra-SCN oscillators, monitored using the pattern of expression of clock gene products, are $180^{\circ}$ out of phase in reference to those of nocturnal rodents $(41,42)$. Interestingly, and pertinent to the discussion of human eveningness, with access to running wheels, some grass rats switch to a predominantly nocturnal display of wheel-running activity. There is evidence of a "compromise" in night-active (NA) grass rats between diurnal tendencies and the display of activity during the normal rest phase of the species. Thus, NA grass rats keep several features of their diurnal profile including the display of frequent sleep episodes and low body temperatures late in the night $(28,43)$ like those seen in DA animals. This interval of sleep is followed by a pre-dawn peak of activity common to both chronotypes (28). Moreover, even though the NA animals sleep more during the day to recover from the sleep debt created by their nocturnal activity, their day-time sleep is fragmented with relatively short sleep-bout lengths (43).

The retention of some diurnal tendencies in NA grass rats may be due to the diverse responses of extra-SCN brain oscillators to the switch to nocturnal activity. Perhaps not surprisingly, the adoption of a NA profile does not affect the phase of clock gene [PERIOD 1 and 2 (PER1/2)] rhythms in the SCN (41) or the nocturnal production of melatonin (36). However, most extra-SCN brain regions that express rhythms in PER 1/2 display a complete reversal of the time of peak expression when grass rats become $\mathrm{NA}$, thus making the circadian profile of NA grass rats similar to that of nocturnal rodents (41). But, the reversal is not universal, again revealing features that are retained by NA grass rats from their antecedent diurnal profile. Outside the hypothalamus, the central amygdala shows a very similar pattern in NA and DA grass rats that contrasts with what is seen in the rest of the extrahypothalamic brain (41). In the extra-SCN hypothalamus (Figure 1), the paraventricular nucleus $(\mathrm{PVN})$ shows a phase reversal in NA grass rats (36), but the ventral subparaventricular zone remains fixed and similar in phase to that of DA animals (41). Most relevant for understanding the sleep fragmentation of NA grass rats during the day is the response of the hypothalamic histaminergic nuclei [i.e., the dorsal and ventral tuberomammillary nuclei (dTMN and VTMN), respectively] to the switch to nocturnality in these animals. Like the PVN, the oscillator of the vTMN of NA grass rats shows a phase reversal, but in sharp contrast that of the dTMN, it retains the phase typical of DA animals (44). These

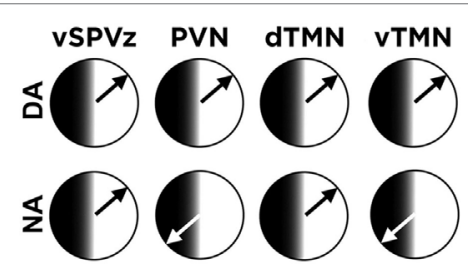

FIGURE 1 | Mosaic of phases of the PERIOD 1 rhythm in hypothalamic extra-suprachiasmatic nucleus (SCN) regions: The phase of the rhythm is similar between day- and night-active (DA and NA, respectively) grass rats for the ventral subparaventricular zone (VZPVz) and the dorsal tuberomammillary nucleus (dTMN). In contrast and similar to most extra-SCN oscillators outside the hypothalamus, the rhythm is $180^{\circ}$ out of phase between DA and NA grass rats in the paraventricular nucleus (PVN) and in the ventral tuberomammillary nucleus (VTMN). See text for references. 
results suggest that the fragmented recovery sleep of NA grass rats results from a mismatch between rhythms in components of the histaminergic arousal system of the tuberomammillary nuclei (45) and the rest/activity cycle. The work with NA and DA grass rats suggests that although temporal phenotypes are flexible and may change in service of energy homeostasis, those changes are not likely to be complete. The compromises between diurnal and nocturnal features of NA grass rats most likely reflect an internal circadian desynchrony that may be an additional cost paid by humans who voluntarily adopt a nocturnal profile. It would be instructive to determine if temporal niche switches in the wild (31) are also associated with similar circadian costs to accompany risks due to exposure to different competitors and/or predators for whom they lack preparation (32).

\section{FUTURE CONSIDERATIONS}

Available models using constrained (forced activity and timed sleep deprivation) or unconstrained (wheel running availability) activity during the natural rest phase of a species do not incorporate the effect of the type of engagement with the environment that goes on during the new active phase. In particular, these models do not replicate instances of human nocturnal activity with significant attentional and cognitive demands, e.g., nurses in hospitals or technicians working at nuclear plants. Experiments in which nocturnal laboratory rats are trained and tested during the day on tasks that demand enhanced attentional performance provide evidence of clear circadian effects that include a shift to a predominantly diurnal chronotype with salient anticipatory activity that persists for days after the training is discontinued $(46,47)$. Activities with low cognitive demands, such as spatial learning or training using operant tasks with low attentional requirements, do not substantially affect circadian activity; neither do daily handling or restriction of water availability to the light phase. $(46,47)$. The circadian effects of high cognitivedemand tasks are likely mediated by cholinergic inputs to the SCN (11), which may affect the nucleus in ways different from other forms of temporal niche switches. Determining the effects on metabolism and energy balance of different activities during

\section{REFERENCES}

1. Megdal SP, Kroenke CH, Laden F, Pukkala E, Schernhammer ES. Night work and breast cancer risk: a systematic review and meta-analysis. Eur J Cancer (2005) 41:2023-32. doi:10.1016/j.ejca.2005.05.010

2. Tucker P, Marquie JC, Folkard S, Ansiau D, Esquirol Y. Shiftwork and metabolic dysfunction. Chronobiol Int (2012) 29:549-55. doi:10.3109/07420528. 2012.675259

3. Sakata K, Suwazono Y, Harada H, Okubo Y, Kobayashi E, Nogawa K. The relationship between shift work and the onset of hypertension in male Japanese workers. J Occup Environ Med (2003) 45:1002-6. doi:10.1097/01. jom.0000085893.98441.96

4. Marquie JC, Tucker P, Folkard S, Gentil C, Ansiau D. Chronic effects of shift work on cognition: findings from the VISAT longitudinal study. Occup Environ Med (2015) 72:258-64. doi:10.1136/oemed-2013-101993

5. Minguez-Alarcon L, Souter I, Williams PL, Ford JB, Hauser R, Chavarro JE, et al. Occupational factors and markers of ovarian reserve and response among women at a fertility centre. Occup Environ Med (2017) 74:426-31. doi:10.1136/oemed-2016-103953 the rest phase, with varied cognitive demands, would add significantly to the value of animal models of human night work or eveningness. Also important to consider when assessing the cost of constrained or unconstrained switches in temporal niche is the influence of circadian phase on cognitive competence. Thus, just like cognitive effort can affect the temporal distribution of activity, time of day can affect the acquisition (46) or retention of learned tasks $(46,48)$. How cognitive functions may be affected by changes in the preferred phase for the display of activity in different animal models represents an important area to explore with respect to the cost of activity during the night, both in night work and in human eveningness.

\section{ETHICS STATEMENT}

The work from our group discussed here was carried out in accordance with the recommendations of the Michigan State University All University Committee on Animal Use and Care, and the National Institute of Health guide for the Care and Use of Laboratory Animals. All protocols were approved by the Michigan State University All University Committee on Animal Use and Care.

\section{AUTHOR CONTRIBUTIONS}

The ideas presented here stem from discussions among the three authors, AN wrote the first draft and LS and LY edited and expanded the initial version.

\section{ACKNOWLEDGMENTS}

We thank Dr. Collen Novak for helpful suggestions on earlier versions of this paper, and Daniel A. Nunez for graphic art work for the figure.

\section{FUNDING}

The work using the Nile grass rat as a model was supported by RO1MH53433 to LS, AN, and D. Weaver.
6. Plano SA, Casiraghi LP, Garcia Moro P, Paladino N, Golombek DA, Chiesa JJ. Circadian and metabolic effects of light: implications in weight homeostasis and health. Front Neurol (2017) 8:558. doi:10.3389/fneur.2017.00558

7. Stephan FK, Zucker I. Circadian rhythms in drinking behavior and locomotor activity of rats are eliminated by hypothalamic lesions. Proc Natl Acad Sci US A (1972) 69:1583-6. doi:10.1073/pnas.69.6.1583

8. Moore RY, Eichler VB. Loss of a circadian adrenal corticosterone rhythm following suprachiasmatic lesions in the rat. Brain Res (1972) 42:201-6. doi:10.1016/0006-8993(72)90054-6

9. Hattar S, Kumar M, Park A, Tong P, Tung J, Yau KW, et al. Central projections of melanopsin-expressing retinal ganglion cells in the mouse. J Comp Neurol (2006) 497:326-49. doi:10.1002/cne.20970

10. Hastings MH, Reddy AB, Maywood ES. A clockwork web: circadian timing in brain and periphery, in health and disease. Nat Rev Neurosci (2003) 4:649-61. doi:10.1038/nrn1177

11. Gritton HJ, Stasiak AM, Sarter M, Lee TM. Cognitive performance as a zeitgeber: cognitive oscillators and cholinergic modulation of the SCN entrain circadian rhythms. PLoS One (2013) 8:e56206. doi:10.1371/journal. pone.0056206 
12. Opperhuizen AL, van Kerkhof LW, Proper KI, Rodenburg W, Kalsbeek A. Rodent models to study the metabolic effects of shiftwork in humans. Front Pharmacol (2015) 6:50. doi:10.3389/fphar.2015.00050

13. Salgado-Delgado R, Angeles-Castellanos M, Buijs MR, Escobar C. Internal desynchronization in a model of night-work by forced activity in rats. Neuroscience (2008) 154:922-31. doi:10.1016/j.neuroscience.2008.03.066

14. Salgado-Delgado R, Angeles-Castellanos M, Saderi N, Buijs RM, Escobar C. Food intake during the normal activity phase prevents obesity and circadian desynchrony in a rat model of night work. Endocrinology (2010) 151:1019-29. doi:10.1210/en.2009-0864

15. Salgado-Delgado RC, Saderi N, Basualdo Mdel C, Guerrero-Vargas NN, Escobar C, Buijs RM. Shift work or food intake during the rest phase promotes metabolic disruption and desynchrony of liver genes in male rats. PLoS One (2013) 8:e60052. doi:10.1371/journal.pone.0060052

16. Barclay JL, Husse J, Bode B, Naujokat N, Meyer-Kovac J, Schmid SM, et al. Circadian desynchrony promotes metabolic disruption in a mouse model of shiftwork. PLoS One (2012) 7:e37150. doi:10.1371/journal.pone.0037150

17. Hsieh WH, Escobar C, Yugay T, Lo MT, Pittman-Polletta B, SalgadoDelgado R, et al. Simulated shift work in rats perturbs multiscale regulation of locomotor activity. J R Soc Interface (2014) 11. doi:10.1098/rsif.2014.0318

18. Leenaars CH, Kalsbeek A, Hanegraaf MA, Foppen E, Joosten RN, Post G, et al. Unaltered instrumental learning and attenuated body-weight gain in rats during non-rotating simulated shiftwork. Chronobiol Int (2012) 29:344-55. doi:10.3109/07420528.2011.654018

19. Cappuccio FP, Taggart FM, Kandala NB, Currie A, Peile E, Stranges S, et al. Meta-analysis of short sleep duration and obesity in children and adults. Sleep (2008) 31:619-26. doi:10.1093/sleep/31.5.619

20. Barf RP, Van Dijk G, Scheurink AJ, Hoffmann K, Novati A, Hulshof HJ, et al. Metabolic consequences of chronic sleep restriction in rats: changes in body weight regulation and energy expenditure. Physiol Behav (2012) 107:322-8. doi:10.1016/j.physbeh.2012.09.005

21. Husse J, Kiehn JT, Barclay JL, Naujokat N, Meyer-Kovac J, Lehnert H, et al. Tissue-specific dissociation of diurnal transcriptome rhythms during sleep restriction in mice. Sleep (2017) 40. doi:10.1093/sleep/zsx068

22. Schmidt S, Randler C. Morningness-eveningness and eating disorders in a sample of adolescent girls. J Individ Differ (2010) 31:38-45. doi:10.1027/ 1614-0001/a000005

23. Yu JH, Yun CH, Ahn JH, Suh S, Cho HJ, Lee SK, et al. Evening chronotype is associated with metabolic disorders and body composition in middleaged adults. JClin Endocrinol Metab (2015) 100:1494-502. doi:10.1210/ jc. 2014-3754

24. McHill AW, Phillips AJ, Czeisler CA, Keating L, Yee K, Barger LK, et al. Later circadian timing of food intake is associated with increased body fat. Am J Clin Nutr (2017) 106:1213-9. doi:10.3945/ajcn.117.161588

25. Arble DM, Bass J, Laposky AD, Vitaterna MH, Turek FW. Circadian timing of food intake contributes to weight gain. Obesity (Silver Spring) (2009) 17:2100-2. doi:10.1038/oby.2009.264

26. Castillo-Ruiz A, Nixon JP, Smale L, Nunez AA. Neural activation in arousal and reward areas of the brain in day-active and night-active grass rats. Neuroscience (2010) 165:337-49. doi:10.1016/j.neuroscience.2009.10.019

27. Castillo-Ruiz A, Nunez AA. Fos expression in arousal and reward areas of the brain in grass rats following induced wakefulness. Physiol Behav (2011) 103:384-92. doi:10.1016/j.physbeh.2011.03.011

28. Blanchong JA, McElhinny TL, Mahoney MM, Smale L. Nocturnal and diurnal rhythms in the unstriped Nile rat, Arvicanthis niloticus. J Biol Rhythms (1999) 14:364-77. doi:10.1177/074873099129000777

29. Vivanco P, Ortiz V, Rol MA, Madrid JA. Looking for the keys to diurnality downstream from the circadian clock: role of melatonin in a dual-phasing rodent, Octodon degus. JPineal Res (2007) 42:280-90. doi:10.1111/j.1600-079X.2007.00418.x

30. van der Veen DR, Riede SJ, Heideman PD, Hau M, van der Vinne V, Hut RA. Flexible clock systems: adjusting the temporal programme. Philos Trans R Soc Lond B Biol Sci (2017) 372. doi:10.1098/rstb.2016.0254

31. Daan S, Spoelstra K, Albrecht U, Schmutz I, Daan M, Daan B, et al. Lab mice in the field: unorthodox daily activity and effects of a dysfunctional circadian clock allele. J Biol Rhythms (2011) 26:118-29. doi:10.1177/0748730410397645
32. Riede SJ, van der Vinne V, Hut RA. The flexible clock: predictive and reactive homeostasis, energy balance and the circadian regulation of sleep-wake timing. J Exp Biol (2017) 220:738-49. doi:10.1242/jeb.130757

33. van der Vinne V, Gorter JA, Riede SJ, Hut RA. Diurnality as an energy-saving strategy: energetic consequences of temporal niche switching in small mammals. J Exp Biol (2015) 218:2585-93. doi:10.1242/jeb.119354

34. Hut RA, Pilorz V, Boerema AS, Strijkstra AM, Daan S. Working for food shifts nocturnal mouse activity into the day. PLoS One (2011) 6:e17527. doi:10.1371/ journal.pone.0017527

35. van der Vinne V, Riede SJ, Gorter JA, Eijer WG, Sellix MT, Menaker M, et al. Cold and hunger induce diurnality in a nocturnal mammal. Proc Natl Acad Sci U S A (2014) 111:15256-60. doi:10.1073/pnas.1413135111

36. Martin-Fairey CA, Ramanathan C, Stowie A, Walaszczyk E, Smale L, Nunez AA. Plastic oscillators and fixed rhythms: changes in the phase of clock-gene rhythms in the PVN are not reflected in the phase of the melatonin rhythm of grass rats. Neuroscience (2015) 288:178-86. doi:10.1016/j. neuroscience.2014.12.040

37. Calisi RM, Bentley GE. Lab and field experiments: are they the same animal? Horm Behav (2009) 56:1-10. doi:10.1016/j.yhbeh.2009.02.010

38. Smale L, Nunez AA, Schwartz MD. Rhythms in a diurnal brain. Biol Rhythm Res (2008) 39:305-18. doi:10.1080/09291010701682666

39. Gaillard F, Bonfield S, Gilmour GS, Kuny S, Mema SC, Martin BT, et al. Retinal anatomy and visual performance in a diurnal cone-rich laboratory rodent, the Nile grass rat (Arvicanthis niloticus). J Comp Neurol (2008) 510:525-38. doi:10.1002/cne.21798

40. Gaillard F, Karten HJ, Sauve Y. Retinorecipient areas in the diurnal murine rodent Arvicanthis niloticus: a disproportionally large superior colliculus. J Comp Neurol (2013) 521:1699-726. doi:10.1002/cne.23303

41. Ramanathan C, Stowie A, Smale L, Nunez AA. Phase preference for the display of activity is associated with the phase of extra-suprachiasmatic nucleus oscillators within and between species. Neuroscience (2010) 170:758-72. doi:10.1016/j.neuroscience.2010.07.053

42. Lambert CM, Weaver DR. Peripheral gene expression rhythms in a diurnal rodent. J Biol Rhythms (2006) 21:77-9. doi:10.1177/0748730405281843

43. Schwartz MD, Smale L. Individual differences in rhythms of behavioral sleep and its neural substrates in Nile grass rats. J Biol Rhythms (2005) 20:526-37. doi:10.1177/0748730405280924

44. Nunez AA, Groves T, Martin-Fairey C, Ramanathan C, Stowie A, Cramm S, et al. The Cost of Nocturnal Activity for a Diurnal Brain. New Orleans, LA: Society for Neuroscience (2012).

45. Castillo-Ruiz A, Gall AJ, Smale L, Nunez AA. Day-night differences in neural activation in histaminergic and serotonergic areas with putative projections to the cerebrospinal fluid in a diurnal brain. Neuroscience (2013) 250:352-63. doi:10.1016/j.neuroscience.2013.07.007

46. Gritton HJ, Sutton BC, Martinez V, Sarter M, Lee TM. Interactions between cognition and circadian rhythms: attentional demands modify circadian entrainment. Behav Neurosci (2009) 123:937-48. doi:10.1037/ a0017128

47. Gritton HJ, Kantorowski A, Sarter M, Lee TM. Bidirectional interactions between circadian entrainment and cognitive performance. Learn Mem (2012) 19:126-41. doi:10.1101/lm.023499.111

48. Martin-Fairey CA, Nunez AA. Circadian modulation of memory and plasticity gene products in a diurnal species. Brain Res (2014) 1581:30-9. doi:10.1016/j.brainres.2014.07.020

Conflict of Interest Statement: The authors declare that the research was conducted in the absence of any commercial or financial relationships that could be construed as a potential conflict of interest.

Copyright (c) 2018 Nunez, Yan and Smale. This is an open-access article distributed under the terms of the Creative Commons Attribution License (CC BY). The use, distribution or reproduction in other forums is permitted, provided the original author(s) and the copyright owner are credited and that the original publication in this journal is cited, in accordance with accepted academic practice. No use, distribution or reproduction is permitted which does not comply with these terms. 